\title{
Nobiletin enhances Pirarubicin chemosensitivity of breast cancer cell MDA-MB-231 by regulating PER2
}

\author{
Fahu Yuan ${ }^{1,2}$, Xinghua Liao ${ }^{1}$, Tongcun Zhang ${ }^{1, *}$ \\ ${ }^{1}$ Institute of Biology and Medicine, College of Life and Health Sciences, Wuhan University of Science and Technology, Wuhan, Hubei, \\ China \\ ${ }^{2}$ School of medicine, Jianghan University, Wuhan, Hubei, China
}

\begin{abstract}
To explore the potential mechanism of nobiletin in improving the sensitivity of breast cancer MDA-MB-231 cells to Pirarubicin. MDA-MB-231 cells were randomly divided into Control group, Nobiletin group, Pirarubicin group and Nobiletin co-Pirarubicin group. CCK-8 assay was used to detect the inhibitory effect of Nobiletin co-Pirarubicin on the proliferation of MDA-MB-231 cells, and the cell cycle and apoptosis of MDA-MB-231 cells were detected by flow cytometry. The effect of nobiletin combined with Pirarubicin on the migration and repair ability of MDA-MB-231 cells was detected by scratch test. Western blotting was used to determine the effect of nobiletin co-Pirarubicin on Period2 (PER2), apoptosisrelated proteins Bax and BCL-2 expression levels in MDA-MB-231 cells. The results showed that the sensitivity of MDA-MB-231 cells to Pirarubicin was enhanced by nobiletin. Compared with Pirarubicin or nobiletin alone, Pirarubicin combined with nobiletin promoted the apoptosis of MDA-MB-231 cells and the expression of apoptosis-related protein Bax, and reduced the expression of apoptosis-inhibiting protein BCL-2. The combination of nobiletin and Pirarubicin significantly inhibited the proliferation and promoted the apoptosis of MDA-MB-231 cells, which may be mediated by the enhancement of PER2 expression by nobiletin.
\end{abstract}

\section{Introduction}

Breast cancer is a kind of solid tumor with high malignancy, insidious onset and strong invasion and metastasis ability. Because the early symptoms are not obvious, most breast cancer patients have missed the best period of surgical resection by the time of diagnosis, and can only be treated by radiotherapy and chemotherapy.

For nearly 20 years, the chemotherapy drug Pirarubicin has been the drug of choice for patients with advanced breast cancer. However, its side effects and easy access to drug resistance have greatly weakened its clinical benefits. In recent years, the research results show that the circadian rhythms protein gene (PER1/2/3) has a strict regulation on cell cycle, normal cell cycle is the Cyclins CDKs-CKIs molecular network system under the precise control of the cell cycle in strict time order along the G1-G2-M-S phase operation. PER2 is generally considered to be a tumor suppressor gene. On the one hand, the overexpression of PER2 significantly reduces the expression of c-MYC and Cyclins, which leads to the arrest of tumor cells in the G1 phase. At the same time, the expression of Weel can be directly upregulated or the expression of CyclinB1 can be inhibited by up-regulating p53, so that cancer cells can be stagnated in the G2 phase [2].
Nobiletin is a kind of natural multifunctional small molecule with anti-tumor, anti-inflammatory and other biological activities. Previous studies have found that nobiletin has not only a highly effective and low-toxicity anti-tumor effect, but also a tumor drug sensitization compound, which, together with the chemotherapy drug gemcitabine and the targeted drug Sorafenib, can produce a good synergistic effect in killing tumor cells. Therefore, it is speculated that the combination of nobiletin and Pirarubicin may also play a synergistic role in killing breast cancer cells. Therefore, the MDA-MB231 cell was selected in this study, and the effects of nobiletin combined with Pirarubicin on proliferation and apoptosis of breast cancer cells, and the mechanism of action of Pirarubicin sensitized by nobiletin was preliminarily explored, so as to provide reference for clinical treatment.

\section{Material and method}

\subsection{Cell lines and reagents}

The human breast cancer cell line MDA-MB-231 was purchased from the Cell Bank of Shanghai, Chinese Academy of Sciences. DMEM high glucose medium, $0.25 \%$ trypsin (including EDTA), fetal bovine serum, penicillin and streptomycin were purchased from Gibco,

\footnotetext{
* Corresponding author: zhangtongcun $@$ wust.edu.cn
} 
BCL-2, Bax, PER2, ACTIN and other primary antibodies were purchased from CST, Sheep anti-rabbit IgG-HRP antibodies were purchased from Santa Cruz Biotechnology Company. Nobiletin and Pirarubicin were purchased from Medchem Express Company.

\subsection{Cell culture}

The MDA-MB-231 cells were cultured in $37{ }^{\circ} \mathrm{C}$ incubators with a $\mathrm{CO}_{2}$ concentration of $5 \%$, and the DMEM high glucose medium containing 10\% FBS was added with $1 \%$ penicillin/streptomycin solution to prevent bacterial contamination. The adherent cells were digestive and subcultured with trypsin with a mass fraction of $0.25 \%$, and subcultured every $2 \sim 3$ days.

\subsection{CCK-8 assay}

MDA-MB-231 cells were seeded in 96-well plates at a density of 5,000 cells per well and cultured for $24 \mathrm{~h}$. Then nobiletin were dissolved in DMSO and continuously diluted in DMEM medium to various concentrations before being added. After the cells and the compound were incubated together for $48 \mathrm{~h}$, the $10 \mu \mathrm{L}$ CCK-8 solution was added to each well and incubated at $37{ }^{\circ} \mathrm{C}$ for 30 minutes. The absorbance value (OD) at the wavelength of $450 \mathrm{~nm}$ was determined by a micrometer. The experiment was repeated three times.

\subsection{Flow cytometry}

MDA-MB-231 cells were seeded in 96-well plates at a density of 5,000 cells per well and cultured for $24 \mathrm{~h}$. After $48 \mathrm{~h}$ of drug treatment, the medium was removed, rinsed with PBS for 2 times, digested with trypsin, centrifuged at $1500 \times \mathrm{g}$ for $5 \mathrm{~min}$, resuspended with PBS, and centrifuged again. After removal of the supernatant, cells were prepared into a suspension with $500 \mu \mathrm{L} 1 \times$ binding buffer and transferred to flow cytometry. Annexin-V $5 \mu \mathrm{L}$ and PI $10 \mu \mathrm{L}$ were added for staining at room temperature and dark for $15 \mathrm{~min}$. The experiment was repeated three times.

\subsection{Western blot}

Logarithmically growing cells were inoculated in a 6well plate $\left(2 \times 10^{5}\right.$ cells/well $)$, adherent overnight culture, medium was changed, DMSO dissolved drugs were added, incubated for $24 \mathrm{~h}$, cells were collected, and soluble proteins were harvested by lysis. The boiled denatured protein samples performed with SDS-PAGE, after the protein electric transfed to the PVDF membrane, fllowed with 5\% skim milk closed 90 min, incubation with corresponding specificity antibody $(1: 1000)$ at room temperature for $2 \mathrm{~h}$, fllowed with $4{ }^{\circ} \mathrm{C}$ incubation overnight, reoccupy HRP labeled-IgG antibodies (1:3, 000) incubation $1.5 \mathrm{~h}$ at room temperature. The ECL solution was added to the film, incubated in anechoic chamber for 1 2 min, and exposed and photographed in chemiluminescence detector, statistic grey value, calculate the relative expression related proteins. The experiment was repeated three times.

\subsection{Statistical analysis}

SPSS 21.0 statistical software was used for statistical analysis of conventional data, Measurement data presented as mean \pm standard deviation. For multiple comparisons, one-way analysis of variance (ANOVA) with Newman-Keuls's post-hoc test was used. A value of $P<0.05$ was regarded as significant.

\section{Results}

\subsection{Nobiletin synergistic enhancement of the sensitivity of breast cancer cells to Pirarubicin}

The survival of human breast cancer cells MDA-MB231 was detected by CCK- 8 method after a 24 h-period administration, so as to determine the inhibition rate of nobiletin on MDA-MB-231 in breast cancer cells. In the experiment, normal proliferation control was set with DMEM medium containing $10 \%$ fetal bovine serum in the same volume. The results showed that Nobiletin with concentration of $25 \mu \mathrm{M}, 50 \mu \mathrm{M}$ and $100 \mu \mathrm{M}$ could significantly inhibit the proliferation of HUMAN breast cancer cells MDA-MB-231, and Nobiletin with concentration of $50 \mu \mathrm{M}$ could significantly inhibit the proliferation of human breast cancer cells $(P<0.01)$.

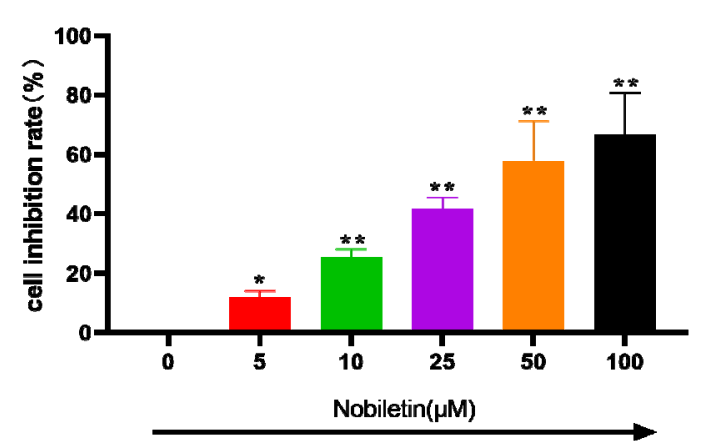

Fig. 1. Inhibition of Nobiletin on proliferation of MDA-MB231. ${ }^{*} P<0.05,{ }^{* *} P<0.01$.

In this study, after pretreatment of MDA-MB-231 with $5 \mu \mathrm{M}$ Pirarubicin, $50 \mu \mathrm{M}$ nobiletin and $5 \mu \mathrm{M}$ Pirarubicin $+50 \mu \mathrm{M}$ nobiletin, and incubation of MDAMB-231 with serum free medium as the control group, the influence of each group on the inhibition of MDAMB-231 was detected after $24 \mathrm{~h}$ of culture. The results showed that the inhibition rates of Nobiletin group and the combined group to MDA-MB-231 cells were $51.69 \%$ $48.24 \%$ and $68.41 \%$, respectively. 


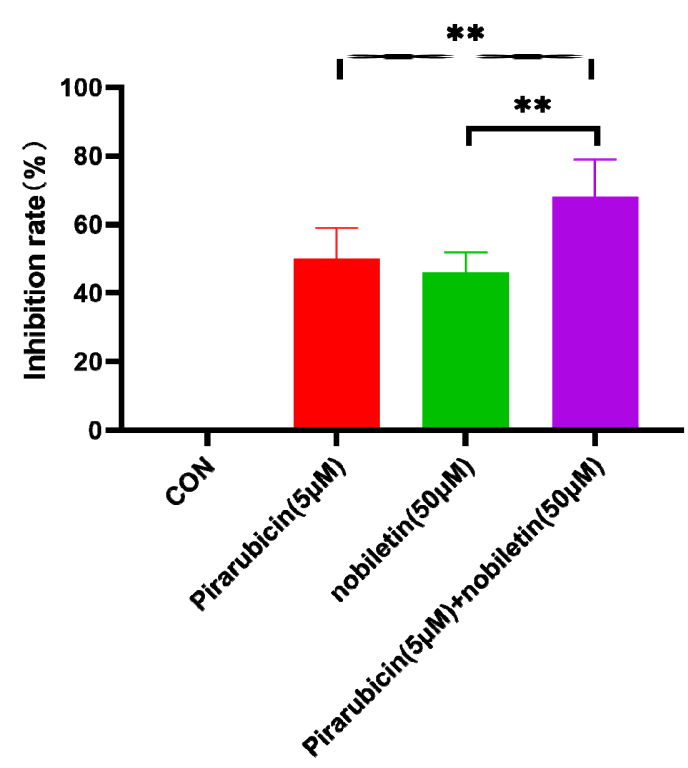

Fig. 2 Nobiletin and Pirarubicin inhibited the proliferation of MDA-MB-231. ${ }^{* * P} P<0.01$.

\subsection{Effect of Nobiletin combined with Pirarubicin on cell cycle of MDA-MB-231}

In order to study the effect of Nobiletin on the cell cycle of MDA-MB-231 induced by Pirarubicin, the cell cycle of MDA-MB-231 was detected by flow cytometry. The results showed that compared with CON group, the cell cycle process of MDA-MB-231 in Pirarubicin group was slowed down, the cell percentage in S phase and G2/M phase increased significantly, and the cell percentage in G0/G1 phase decreased significantly. Compared with the Pirarubicin group, the cell cycle of MDA-MB-231 in the Nobiletin+Pirarubicin group was significantly slowed down, the cell percentage in the S stage and G2/M stage increased to different degrees, and the cell percentage in the $\mathrm{G} 0 / \mathrm{G} 1$ stage decreased to different degrees.
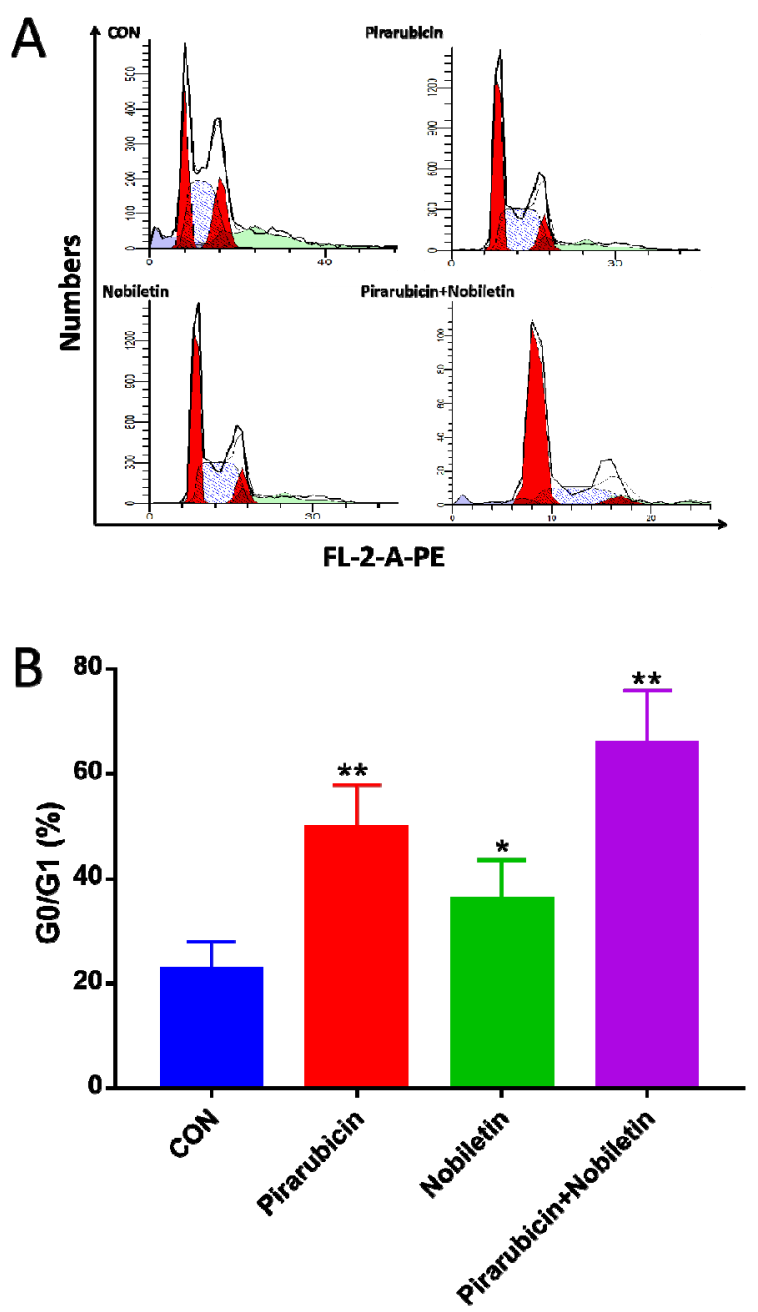

Fig. 3. Influence of Nobiletin and Pirarubicin on the cell cycle of MDA-MB-231.

\subsection{Nobiletin combined with Pirarubicin increased apoptosis of MDA-MB-231 cells}

The flow cytometry results showed that Pirarubicin had a certain effect on apoptosis of breast cancer cells, while Nobiletin alone with $50 \mu \mathrm{mol} / \mathrm{L}$ had a very weak effect on apoptosis, but the combination of Nobiletin and Pirarubicin significantly increased the apoptosis of breast cancer cells(Fig. 4). 


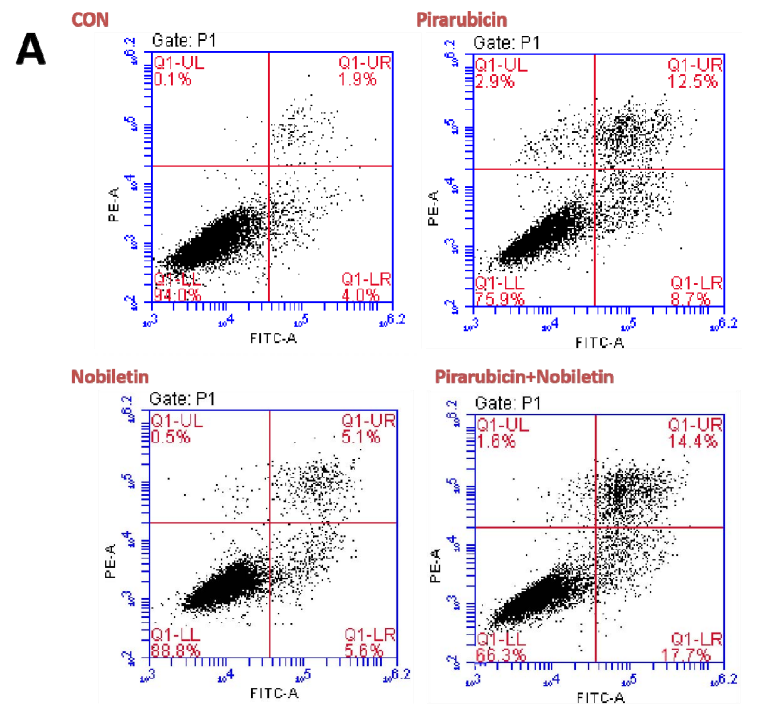

B

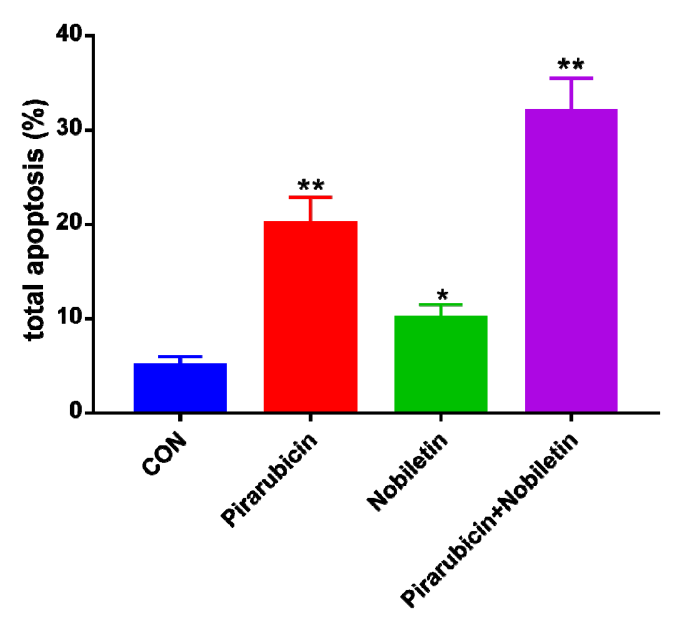

Fig. 2. Effects of Nobiletin and Pirarubicin on apoptosis of MDA-MB-231 cells

\subsection{Nobiletin inhibited the activation of PER2- signaling pathway}

Western blot test showed that the expression of PER2 in cells was increased by Nobiletin. Compared with the single use of Pirarubicin or Nobiletin, the combination of Pirarubicin and Nobiletin promoted the expression of apoptosis-related protein BAX in MDA-MB-231 cells, and reduced the expression of apoptosis-inhibiting protein BCL-2.

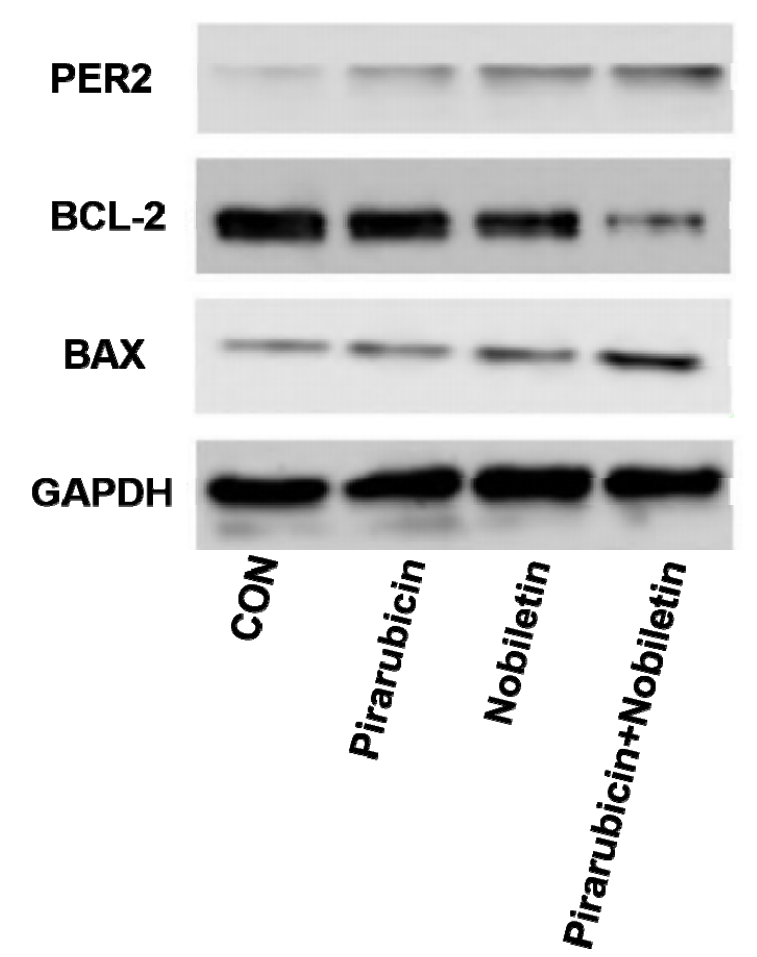

Fig. 3. Effects of Nobiletin and Pirarubicin on PER2 and cyclin related protein expression in MDA-MB-231 cells.

\section{Conclusion}

In this study, CCK-8, flow cytometry and Western blot were used to study the effect of Nobiletin combined with Pirarubici on the biological behavior of human breast cancer MDA-MB-231 cells, and the biological mechanism was preliminarily discussed. The experimental results showed that Nobiletin inhibited the proliferation of MDA-MB-231 cells, blocked the cell cycle, and increased the number of apoptotic cells and the proportion of early/late apoptotic cells by CCK-8 and flow cytometry detection. The above effects of Nobiletin combined with Pirarubicin group were superior to those of Pirarubicin group alone, indicating that Nobiletin has anti-breast cancer effect and can sensitize Pirarubicin. Western blot showed that Nobiletin up-regulated PER2 level in MDA-MB-231 cells, suggesting that per2-cell cycle signaling pathway may mediate the anti-breast cancer process of Nobiletin.

In conclusion, Nobiletin may enhance the anti-tumor effect of Pirarubicin by restoring PER2 signaling pathway.

\section{Acknowledgments}

This project is funded by Grants from the Guiding Project in Science Research Program from Education Department of Hubei (B2020222).

\section{References}

1. Oh Ju-Hee,Lee Joo Hyun,Han Dong-Hee et al. Circadian Clock Is Involved in Regulation of Hepatobiliary Transport Mediated by Multidrug 
Resistance-Associated Protein 2.[J] .J Pharm Sci, 2017, 106: 2491-2498.

2. Hill Steven M,Belancio Victoria P,Dauchy Robert T et al. Melatonin: an inhibitor of breast cancer.[J] .Endocr Relat Cancer, 2015, 22: R183204.

3. Ozkan Asuman Deveci,Sarihan Mehmet,Kaleli Suleyman,In VitroEvaluation of the Effects of Nobiletin on Toll-Like Receptor 3 Signaling Pathways in Prostate Cancer .[J] .Nutr Cancer, 2020, undefined: 1-7.

4. Ashrafizadeh Milad,Zarrabi Ali,Saberifar Sedigheh et al. Nobiletin in Cancer Therapy: How This Plant Derived-Natural Compound Targets Various Oncogene and Onco-Suppressor Pathways. [J]. Biomedicines, 2020, 8.

5. $\mathrm{Xu}$ Yitong, Rogers Connie J,Physical Activity and Breast Cancer Prevention: Possible Role of Immune Mediators.[J] .Front Nutr, 2020, 7: 557997. 